\title{
Electron Trapping in Buried Oxides During Irradiation at 40 and $300 \mathrm{~K}$
}

\author{
R. E. Stahlbush \\ Naval Research Laboratory, Code 6816 \\ Washington, DC 20375
}

\begin{abstract}
The formation of electron traps has been studied in thermally grown silicon dioxide that was encapsulated by polysilicon and annealed at temperatures from 1100 to $1325^{\circ} \mathrm{C}$. Samples with oxide thicknesses of 100 and $400 \mathrm{~nm}$ were examined by cryogenic and room temperature detrapping measurements. Two distinct electron traps were observed in buried oxides; the thermal trap depths are 0.5 and $1.1 \mathrm{eV}$ and the tunneling depths are 0.9 and $1.5 \mathrm{eV}$. The deeper electron trap is filled by room temperature irradiation. The deeper trap is detrapped by thermal excitation between room temperature and $120^{\circ} \mathrm{C}$ or by tunneling at fields between 4 and $5 \mathrm{MV} / \mathrm{cm}$. The formation of both electron traps is diffusion limited and the $\mathrm{Si} / \mathrm{SiO}_{2}$ interfaces are the source or sink for the diffusing species. While shallow trap formation monotonically increases with annealing temperature, trapping in the deeper trap peaks at about $1200^{\circ} \mathrm{C}$ for the $400 \mathrm{~nm}$ thickness and at $1100^{\circ} \mathrm{C}$ or lower for the $100 \mathrm{~nm}$ thickness. The occupation of shallow traps during cryogenic irradiation decreases the occupation of the deeper trap compared with room temperature irradiation. At high concentrations, the shallow traps decrease the tunneling field of the deeper trap by trap-assisted tunneling.
\end{abstract}

\section{INTRODUCTION}

Silicon-on-Insulator (SOI) technologies are being pursued for advanced integrated circuits. Integrated circuits fabricated with SOI wafers offer advantages for radiation-hardening and for low-power operation. The leading methods for SOI wafer fabrication are separation by implantation of oxygen (SIMOX) and the various wafer bonding techniques. All of these technologies require annealing of the buried oxide. The SIMOX fabrication process involves a high temperature anneal $\left(>1300^{\circ} \mathrm{C}\right)$ after oxygen implantation. Wafer bonding techniques also normally anneal the buried oxide after bonding and the annealing temperature is often $1100^{\circ} \mathrm{C}$ or higher. The importance of SOI has generated an interest in understanding defects in the buried oxide and the effects of annealing silicon dioxide that is sandwiched between silicon layers[1-15]. Much of the past work has focused on defects produced by the higher annealing temperature required for SIMOX fabrication. A high concentration of $E^{\prime}$ centers is measured in SIMOX material[7]. Annealing a buried oxide at $1300^{\circ} \mathrm{C}$ has been shown to form a high concentration of deep hole and shallow electron traps [8]. A deeper electron trap has also been observed after cryogenic irradiation [15].

In this paper, the deeper electron trap is studied in more detail. A range of annealing temperatures from 1100 to $1325^{\circ} \mathrm{C}$ is examined with oxide thicknesses of 100 and $400 \mathrm{~nm}$. It is shown that this deeper trap is occupied by room temperature irradiation and it is thermally depopulated between room temperature and $120^{\circ} \mathrm{C}$. The tunneling depth of the trap is $1.5 \mathrm{eV}$ and its thermal depth is $1.1 \mathrm{eV}$. As is the case for the shallow electron trap, the formation of this deeper electron trap appears to be diffusion limited with the $\mathrm{Si} / \mathrm{SiO}_{2}$ interfaces of the buried oxide being the source or sink of the diffusing species. While trapping in the shallow traps either increases or saturates with increasing annealing temperature, trapping in the deeper trap goes through a peak. The maxirnum trapping is near $1200^{\circ} \mathrm{C}$ for the $400 \mathrm{~nm}$ oxide and at $1100^{\circ} \mathrm{C}$ or lower for the $100 \mathrm{~nm}$ samples. In the $400 \mathrm{~nm}$ oxides annealed at $1250^{\circ} \mathrm{C}$ or higher, the concentration of shallow traps is sufficiently high to produce trap-assisted tunneling from the deeper trap. Instead of tunneling directly into the oxide conduction band, electrons tunnel via an empty intermediate shallow trap to the conduction band. At the transition from direct to trap assisted tunneling, an order-of-magnitude calculation of the concentration of shallow traps gives $10^{19} / \mathrm{cm}^{3}$.

This work was supported by the Office of Naval Research. 


\section{EXPERIMENTAL DETAILS}

To investigate the effects of annealing a buried thermal oxide, silicon wafers with different oxide thicknesses and annealing temperatures were prepared. Results from two thicknesses are reported: 100 and 400 $\mathrm{nm}$. The oxides were grown in steam at $850^{\circ} \mathrm{C}$ on $(100)$ 3-4 ohm-cm n-type silicon wafers and encapsulated with $450 \mathrm{~nm}$ of polysilicon and $300 \mathrm{~nm}$ of TEOS deposited oxide. Wafers of each thickness were annealed at temperatures of $1100,1175,1250$ and $1325^{\circ} \mathrm{C}$ using a standard SIMOX cycle, 5 hours in argon plus $1 \%$ oxygen. Following the anneal, the TEOS oxide was removed and the polysilicon was doped n-type by diffusion. Aluminum was deposited on the polysilicon and capacitors were formed by patterning the polysilicon/aluminum layers.

Charge trapping was measured by the cryogenic detrapping technique [8]. With this technique it is possible to independently measure hole and electron trapping after irradiation. The $\mathrm{x}$-ray irradiations were performed at $40 \mathrm{~K}$ or $300 \mathrm{~K}$, room temperature; using a tungsten target tube operated at $30 \mathrm{kV}$. The dose rate was $1.7 \mathrm{krad}\left(\mathrm{SiO}_{2}\right) / \mathrm{s}$ and the total dose was typically $100 \mathrm{krad}\left(\mathrm{SiO}_{2}\right)$. The capacitors were grounded during irradiation. This is normally the most favorable bias condition to investigate electron trapping. The field within the oxide due to the net positive charge provides a potential well that tends to retain electrons until they are captured [15]. The net charge in the oxide was monitored by high frequency capacitor-voltage (C-V) measurements. The voltage shifts of the $\mathrm{C}-\mathrm{V}$ curves were determined by a least square fitting procedure [15].

\section{RESULTS}

The presence of two electron traps with distinctly different trap depths is shown in Fig. 1 using results from the $400 \mathrm{~nm}$ buried oxide annealed at $1175^{\circ} \mathrm{C}$. The electron traps are populated by a radiation dose of 100 $\operatorname{krad}\left(\mathrm{SiO}_{2}\right)$ at $40 \mathrm{~K}$ with the gates grounded and are depopulated by thermal excitation and field-induced tunneling. Following irradiation the electron traps are emptied by a two step process. First, isochronal anneals of 20 minutes from 120 to $400 \mathrm{~K}$ were performed with the capacitors grounded, and second, after returning to $40 \mathrm{~K}$, electrons were removed by field-induced tunneling at electric fields between 0 to $6 \mathrm{MV} / \mathrm{cm}$. A family of curves, one for each annealing temperature plus one without the annealing step is plotted. Each curve shows the negative $\mathrm{C}-\mathrm{V}$ shifts as the net positive

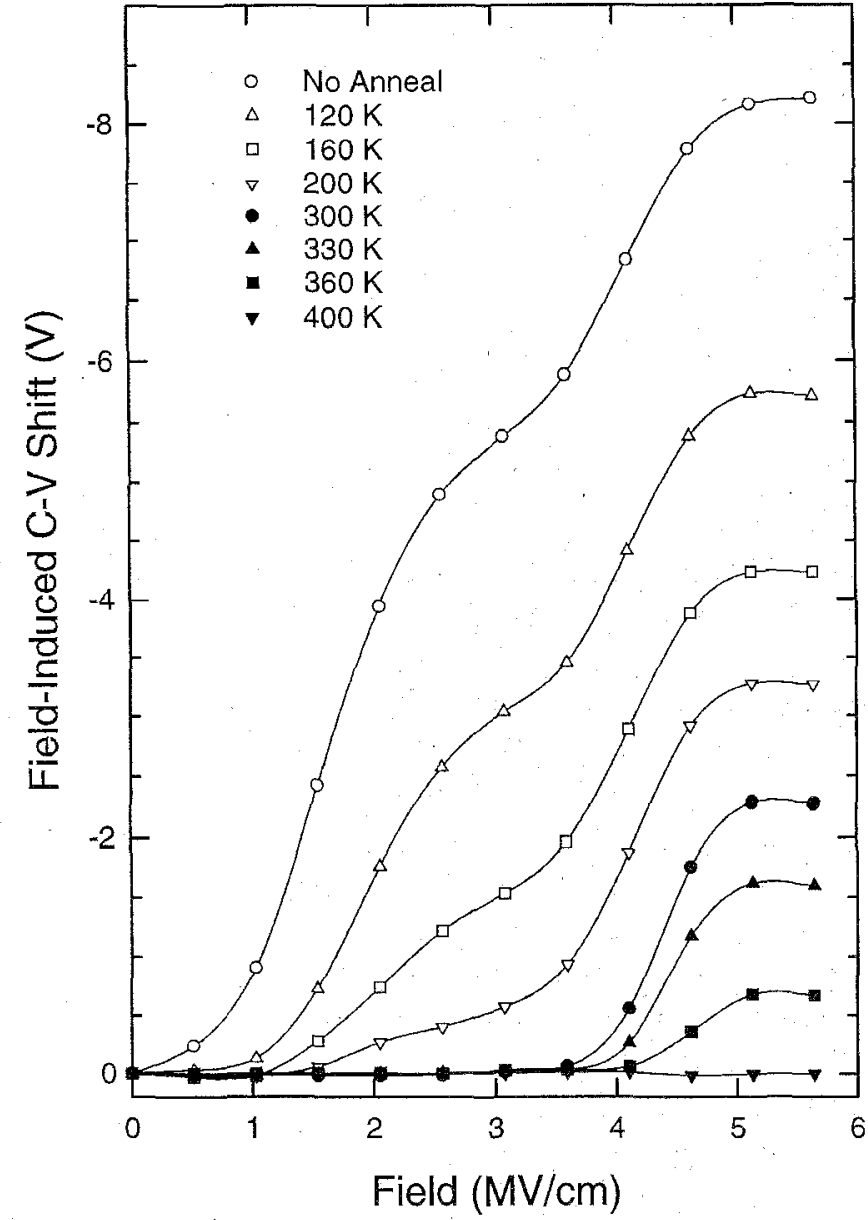

Fig. 1. Field-induced electron detrapping at $40 \mathrm{~K}$. Results from the $400 \mathrm{~nm}$ buried oxide annealed at $1175^{\circ} \mathrm{C}$ are shown. Electron (and hole) traps are populated by a radiation dose of 100 $\mathrm{krad}\left(\mathrm{SiO}_{2}\right)$ with the capacitor gates grounded at $40 \mathrm{~K}$. After the irradiation and before field-induced detrapping a $20 \mathrm{~min}$. thermal detrapping step was inserted at temperatures ranging from $120 \mathrm{~K}$ to $400 \mathrm{~K}\left(123^{\circ} \mathrm{C}\right)$. The top curve was collected without the thermal detrapping step.

charge increases due to electron detrapping by fieldinduced tunneling. Many of the measurements were repeated with two samples and the variations were smaller than the size of the data points in the figure.

The largest positive charge gain is evident when there is no annealing step. While the number of electrons trapped is less than the number of holes trapped, the number of electrons and holes captured are comparable. After irradiation, the $\mathrm{C}-\mathrm{V}$ shift from the net charge is $-5 \mathrm{~V}$. Without the annealing step, the trapped electrons exhibit an $8 \mathrm{~V}$ shift. Thus, the number of trapped electrons is about $60 \%$ of the number of trapped holes.

Without the annealing step, all of the detrapped electrons are removed by tunneling and are swept from 
the buried oxide by the applied field. During the isochronal annealing step with the capacitors grounded, electrons that are thermally excited from traps recombine with trapped holes. After irradiation, there is a net positive charge, and in the absence of an applied field, electrons that are thermally excited from traps move within the potential well formed by the positive charge until they recombine with a trapped hole. The net charge is unchanged as is verified by the negligible $\mathrm{C}-\mathrm{V}$ shift caused by annealing $(<0.1 \mathrm{~V})$. (In annealing measurements made under bias, electrons were removed from the oxide and the net positive charge increased. In the measurements shown in Fig. 1, annealing with the gates grounded was used to minimize the internal field generated by the net positive charge.) After annealing, electrons that remain in traps are removed by tunneling. As the isochronal annealing temperature is increased, more electrons are detrapped by thermal excitation and fewer electrons are detrapped by the applied field.

Both the field and temperature dependencies shown in Fig. 1 show the presence of two electron traps. In the field-induced detrapping there are two distinct steps: one below $3.5 \mathrm{MV} / \mathrm{cm}$ and one above that field. The height of each step is proportional to the number of electrons in each trap. In progressing from the data without annealing to the $300 \mathrm{~K}$ data, the number of electrons in

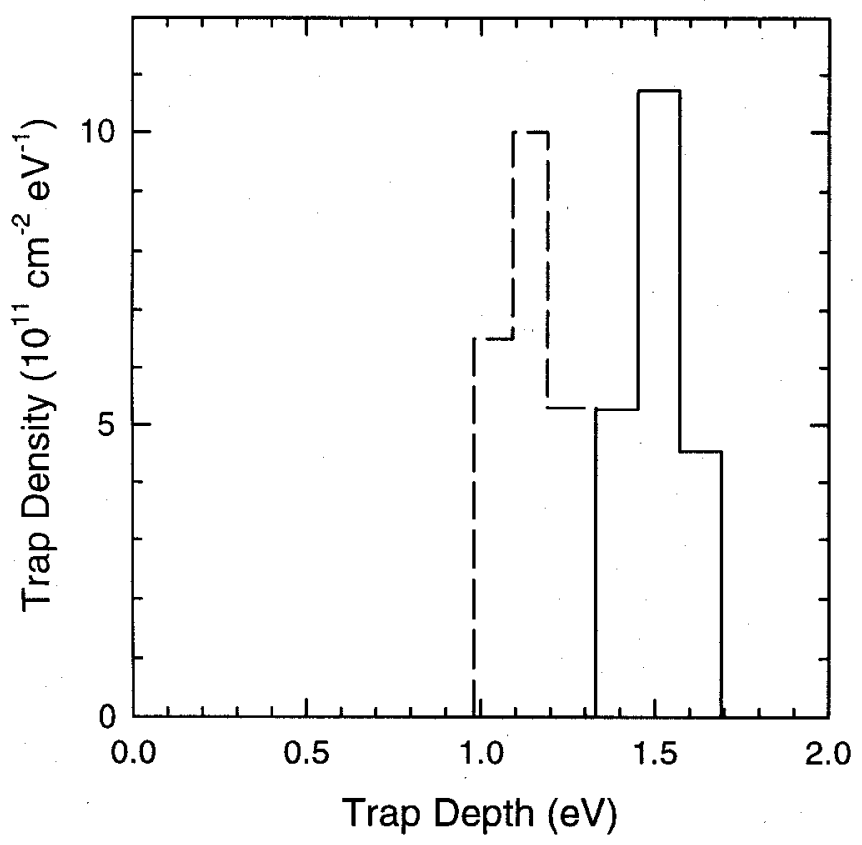

Fig. 2. Trap densities of the deeper electron trap calculated from the data shown in Fig. 1. The trap depth is given with respect to the oxide conduction band. The thermal distribution of trap depths is plotted with a dashed line and the tunneling distribution is shown with the solid line. The average thermal depth is $1.1 \mathrm{eV}$ and the average tunneling depth is $1.5 \mathrm{eV}$. shallow traps after annealing steadily decreases to a negligible number while the step height above 3.5 $\mathrm{MV} / \mathrm{cm}$ is not changed. This shows that electrons are not thermally excited out of the deeper trap at $300 \mathrm{~K}$, room temperature, and below. Between 300 and $400 \mathrm{~K}$, electrons are thermally excited out of the deeper trap, and at $400 \mathrm{~K}\left(\sim 120^{\circ} \mathrm{C}\right)$ electrons are completely removed thermally.

Both of the electron traps are bulk traps in the oxide, and neither responds to the surface potential at the $\mathrm{Si} / \mathrm{SiO}_{2}$ interfaces. The detrapping shown in Fig. 1 is driven by the field inside the oxide and is the same for positive and negative gate voltages. There is a slight difference in the magnitude of the field at which detrapping occurs, but this is due to the contribution of charges in the oxide to the internal oxide field [8]. Thus, these traps are very different from slow states or border traps that do respond to the silicon surface potential [16, 17].

From the data in Fig. 1, the thermal and tunneling trap depths of the two electron traps can be calculated. The correspondence between the thermal trap depth and the temperature at which the trap is emptied is given by [18]

$$
E_{t}=k T \ln [v t]
$$

where $\mathrm{k}$ is the Boltzmann constant, $\mathrm{T}$ is the temperature in Kelvin, $t$ is the annealing time and $v=$ $3 \times 10^{13}\left[(\mathrm{~T} / 300)^{2}\right]$ is the attempt frequency [19]. Similarly, the distribution of tunneling trap depths is calculated by solving the equation [20]

$$
\begin{gathered}
\mathrm{E}_{\mathrm{t}}^{3 / 2} /(\mathrm{qF})=\mathrm{B} \ln \left[\mathrm{AqFt} / \mathrm{E}_{\mathrm{t}}\right] \\
\mathrm{A}=\pi^{2} \mathrm{mqW} \mathrm{W}^{2} / \mathrm{h}^{3} \\
\mathrm{~B}=3 \mathrm{hq} /\left(8 \pi[2 \mathrm{~m}]^{1 / 2}\right)
\end{gathered}
$$

where $F$ is the electric field, $q$ is the electron charge, $t$ is the time at the applied field, $m$ is the electron mass, $h$ is Planck's constant and $\mathrm{W}^{2}=10^{-24} \mathrm{eV}^{2}-\mathrm{cm}^{3}$ is the square of the tunneling matrix element [21].

The thermal and tunneling trap depth distributions of the shallow trap have already been determined and shown in Ref. [8]. The average thermal depth is $0.5 \mathrm{eV}$ and the average tunneling depth is $0.9 \mathrm{eV}$. (In Ref. [8] an older value of the attempt frequency was used $[20$, $21], v=10^{10}\left[(\mathrm{~T} / 300)^{2}\right]$, yielding a thermal trap depth of $0.4 \mathrm{eV}$.) The trap densities of the deeper electron trap are shown in Fig. 2. The thermal depth distribution is shown with the dashed line and a solid line is used for 
the tunneling depth distribution. The average thermal depth is $1.1 \mathrm{eV}$ and the average tunneling depth is 1.5 $\mathrm{eV}$. Note that energy spread of the deeper trap is less than the spread of the shallow trap. This can also be seen by examining Fig. 1. The detrapping field of the shallow trap ranges from 0.5 to $3.5 \mathrm{MV} / \mathrm{cm}$, whereas the deeper trap is fully detrapped between 3.5 and 5 $\mathrm{MV} / \mathrm{cm}$. The thermal detrapping has the same trend. Thermal detrapping of the shallow trap occurs over the temperature range spanning 120 to $300 \mathrm{~K}$. In comparison, the deeper trap is thermally depopulated in the 300 to $400 \mathrm{~K}$ range.

In Fig. 3 the occupation and field-induced detrapping of the deeper electron trap is compared for irradiation and detrapping temperatures of $40 \mathrm{~K}$ and room temperature. Results from the $400 \mathrm{~nm}, 1175^{\circ} \mathrm{C}$ sample set are shown. The open diamonds and open circles show data from room temperature irradiation. The dose was $100 \mathrm{krad}\left(\mathrm{SiO}_{2}\right)$ with the capacitor gates grounded. Following irradiation, electrons were removed by field-induced detrapping either at room temperature, open diamonds, or at $40 \mathrm{~K}$, open circles. Detrapping at both temperatures yields the same number of trapped electrons. However, detrapping at room temperature occurs at fields that are $2 \mathrm{MV} / \mathrm{cm}$ lower. At room temperature, electrons leave traps by the combination of thermal excitation and tunneling. Thus, the apparent tunneling depth at room temperature is lowered. For calculating the trap tunneling depth, this illustrates the importance of performing the fieldinduced detrapping at a sufficiently low temperature to prevent thermal excitation.

A comparison of Fig. 3 with Fig. 1 shows that after a room temperature irradiation the deeper electron trap is occupied, but the shallow electron trap is not occupied. The solid circles replot the results from Fig. 1 for the $300 \mathrm{~K}$ anneal. For both the open and solid circle data sets, there is not any detrapping in the field range of the shallow trap. Both curves have the same field dependence of the deeper electron trap. However, after the room temperature irradiation there are $50 \%$ more electrons trapped in the deeper trap compared with $40 \mathrm{~K}$ irradiation. At $40 \mathrm{~K}$, both traps capture electrons causing fewer electrons to be captured in the deeper trap. Also note that the number of electrons trapped at room temperature is a significant fraction of the number of trapped holes. The net positive charge after either room temperature or $40 \mathrm{~K}$ irradiation is the same and yields a $-5 \mathrm{~V} \mathrm{C}-\mathrm{V}$ shift. Thus, the number of electrons captured in the deeper trap during the room temperature and $40 \mathrm{~K}$ irradiations are $42 \%$ and $32 \%$ of the number of trapped holes, respectively. Not only is there significant

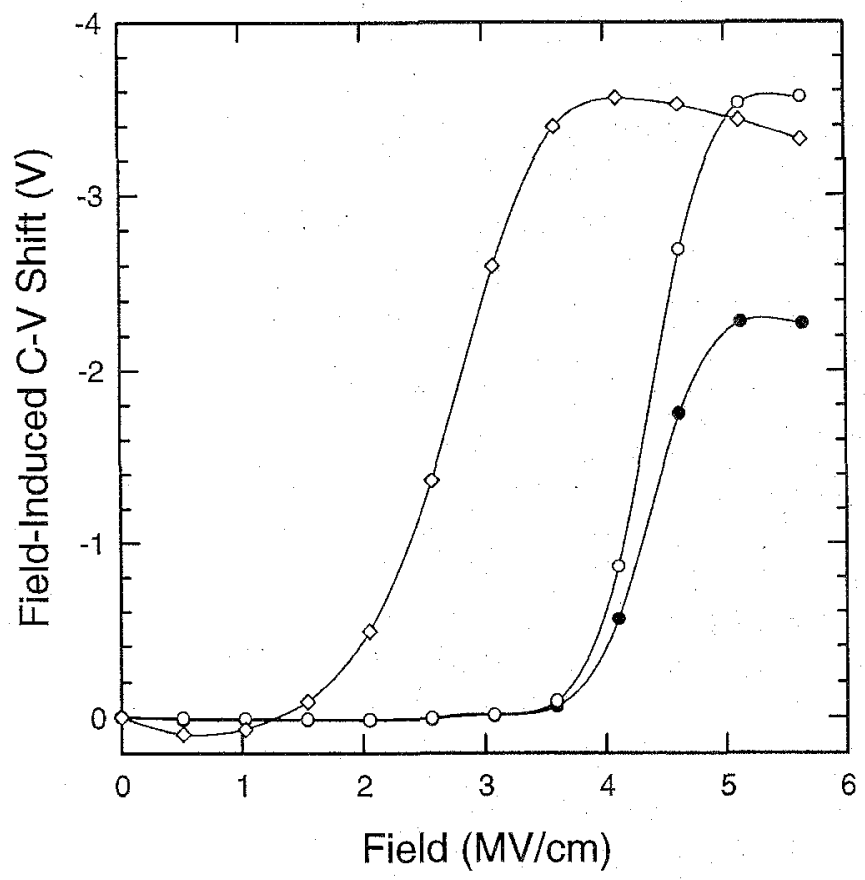

Fig. 3. Field-induced electron detrapping from the deeper electron trap. Results from the $400 \mathrm{~nm}$ buried oxide annealed at $1175^{\circ} \mathrm{C}$ are shown. Electron (and hole) traps are populated by a radiation dose of $100 \mathrm{krad}\left(\mathrm{SiO}_{2}\right)$ with the capacitor gates grounded. The irradiation temperature was $300 \mathrm{~K}$ for the open diamonds and open circles and $40 \mathrm{~K}$ for the closed circles. The temperature of the field-induced detrapping was $300 \mathrm{~K}$ for the open diamonds and $40 \mathrm{~K}$ for the open and closed circles. After the $40 \mathrm{~K}$ irradiation and before the $40 \mathrm{~K}$ field stress of the closed circle data, a 20 minute, $300 \mathrm{~K}$ thermal detrapping step was inserted to depopulate the shallow electron traps.

electron trapping in these buried oxides, but the amount of trapping in any of the traps is affected by the irradiation temperature.

The effect of the oxide annealing temperature on electron trapping during room temperature irradiation is shown in Figs. 4 and 5. The dose was $100 \mathrm{krad}\left(\mathrm{SiO}_{2}\right)$ with the capacitor gates grounded, and the field-induced detrapping was performed at $40 \mathrm{~K}$. Results are shown for 400 and $100 \mathrm{~nm}$ samples in Figs. 4 and 5 , respectively. In Fig. 4, the annealing temperature ranges from 1100 to $1325^{\circ} \mathrm{C}$. Also included are data for samples that were not annealed after polysilicon deposition. Without the annealing step no electron trapping is observed. All of the annealed samples exhibit electron trapping in the deeper electron trap with the maximum trapping occurring at $1175^{\circ} \mathrm{C}$. In contrast, electron trapping in the shallow traps monotonically increases over this annealing temperature range for $400 \mathrm{~nm}$ samples $[8,15]$.

Room temperature electron trapping peaks at a lower annealing temperature for the $100 \mathrm{~nm}$ oxides as 


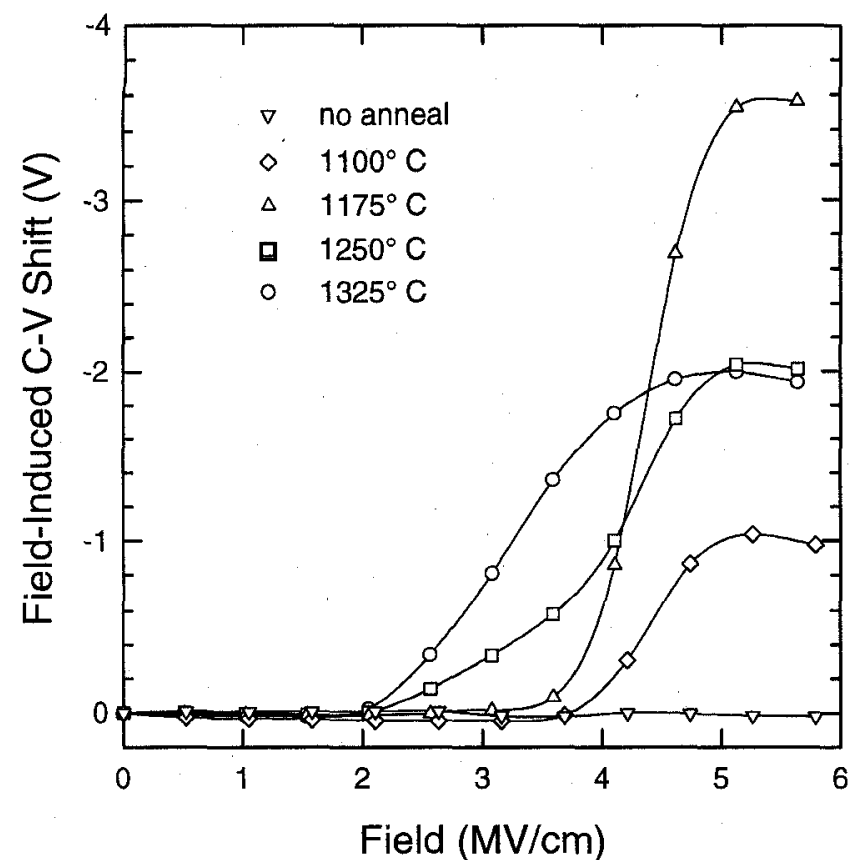

Fig. 4. Field-induced electron detrapping at $40 \mathrm{~K}$ from the deeper electron trap. Electron (and hole) traps are populated by a radiation dose of $100 \mathrm{krad}\left(\mathrm{SiO}_{2}\right)$ with the capacitor gates grounded at room temperature. Results from the $400 \mathrm{~nm}$ buried oxide with annealing temperatures from 1100 to $1325^{\circ} \mathrm{C}$ are shown. Samples that were not annealed are also plotted.

shown in Fig. 5. There is significantly more electron trapping at $1100^{\circ} \mathrm{C}$ than at $1175^{\circ} \mathrm{C}$. The downturn of the curves above $5 \mathrm{MV} / \mathrm{cm}$ is due to electron injection which then neutralizes trapped holes [8]. There is a slight upturn in the $1175^{\circ} \mathrm{C}$ data above $3 \mathrm{MV} / \mathrm{cm}$ suggesting electrons are being detrapped, but this small voltage shift is near the detection limit of the measurements. The $100 \mathrm{~nm}$ buried oxides exhibit the same pattern of shallow versus deeper electron trapping. Shallow electron trapping in the $100 \mathrm{~nm}$ oxides increases from 1100 to $1175^{\circ} \mathrm{C}$ and then saturates [15]. In contrast, trapping in the deeper trap is at a maximum at $1100^{\circ} \mathrm{C}$ or lower.

Previous work has suggested that the formation of the shallow electron traps [15] and of E' centers [14] is diffusion limited and that the $\mathrm{Si} / \mathrm{SiO}_{2}$ interfaces of the buried oxide are the source or sink for the diffusing species. The dependence of electron trapping in the deeper trap on the oxide annealing temperature and oxide thickness suggests that its formation is also diffusion limited. The deeper electron trap has the same relationship between temperature and thickness as the shallow trap. The trapping in shallow traps saturates at a lower temperature for the $100 \mathrm{~nm}$ oxide than for the $400 \mathrm{~nm}$ oxide [15]. Similarly, the maximum trapping in

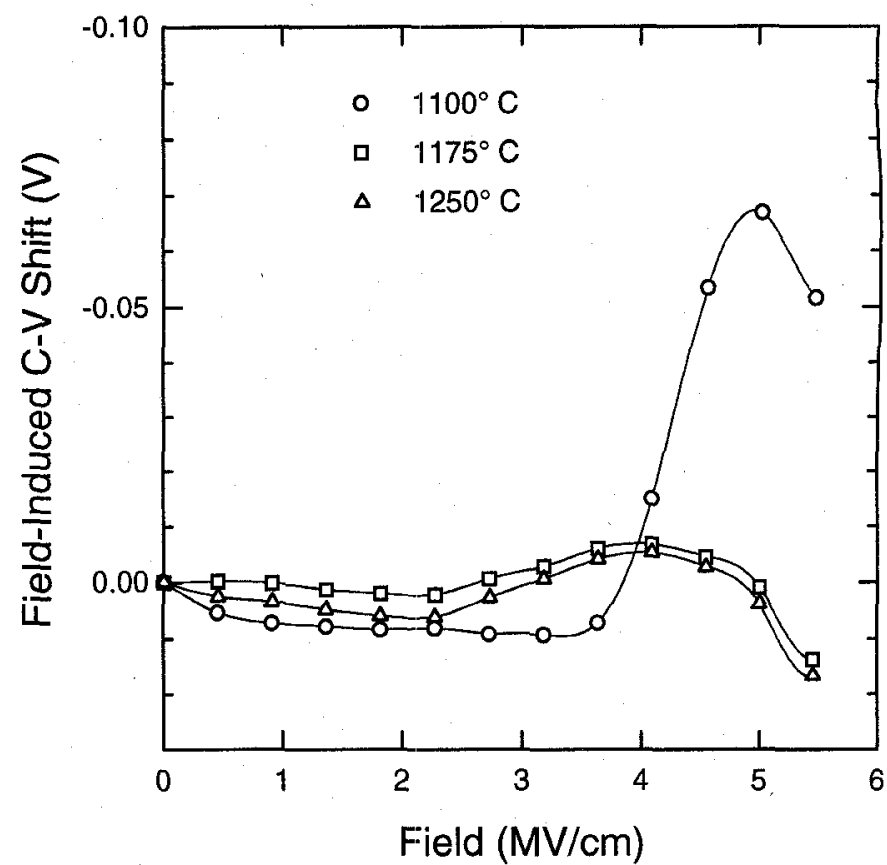

Fig. 5. Field-induced electron detrapping at $40 \mathrm{~K}$ from the deeper electron trap. Results from the $100 \mathrm{~nm}$ buried oxide with annealing temperatures from 1100 to $1250^{\circ} \mathrm{C}$ are shown. Electron (and hole) traps are populated by a radiation dose of 100 $\operatorname{krad}\left(\mathrm{SiO}_{2}\right)$ with the capacitor gates grounded at room temperature.

the deeper trap also occurs at a lower annealing temperature for the $100 \mathrm{~nm}$ buried oxide than for the $400 \mathrm{~nm}$ oxide.

\section{DISCUSSION}

\section{A. Differentiating Electron Detrapping vs. Hole Motion}

Capacitance-voltage measurements are only sensitive to the net charge in the oxide. Furthermore, their sensitivity is weighted toward the $\mathrm{Si} / \mathrm{SiO}_{2}$ interface at which the silicon surface potential is being swept. The relationship between the voltage shift of the C-V curve and the changes in oxide charge are given by

$$
\Delta \mathrm{V}=(\Delta \mathrm{Q} / \mathrm{C})(\mathrm{x} / \mathrm{l})
$$

where $\Delta \mathrm{Q}$ is the change in the charge per area, $\mathrm{C}$ is the capacitance per area, 1 is the oxide thickness and $\mathrm{x}$ is the distance between the gate and the charge. Thus, charges very near the $\mathrm{Si} / \mathrm{SiO}_{2}$ interface are fully measured, charges very near the gate are not detected, voltage shifts for charges at the oxide midpoint are half as large, etc. 
Despite these measurement limitations, it is possible to differentiate electron versus hole detrapping and/or motion. For example, consider the field-induced $\mathrm{C}-\mathrm{V}$ shifts after room temperature irradiation such as shown in Fig. 4. Fields in the 4 to $5 \mathrm{MV} / \mathrm{cm}$ range increase the measured net positive charge in the oxide. Furthermore, the same charge increase is measured for either positive or negative gate polarity. This combination of measurements excludes explanations involving hole detrapping and/or motion within the oxide. The net measured charge increases. Thus, if holes were responsible, they would have to move closer to the $\mathrm{Si} / \mathrm{SiO}_{2}$ interface without leaving the oxide. With a positive gate voltage, it would be possible for holes to be detrapped, move toward the $\mathrm{Si} / \mathrm{SiO}_{2}$ interface and be captured in deeper traps. However, the negative bias results preclude any such explanations.

The thermal excitation behavior is also inconsistent with hole-based explanations. After a cryogenic irradiation, the voltage shift caused by the isochronal annealing step is negligible. If holes were thermally detrapped; they would have to recombine with trapped electrons to maintain the same charge in the oxide. However, that is inconsistent with the observation, that the net charge increases when a bias is applied during the annealing step. Thus, by carefully employing combinations of thermal and applied field steps after irradiation, it is possible to differentiate between detrapping of electrons and holes.

\section{B. Trap-Assisted Tunneling}

At high concentrations of shallow electron traps a significant fraction of the tunneling from the deeper electron trap occurs by trap-assisted tunneling. Instead of tunneling directly from the trap to the oxide conduction band, the electron first tunnels to a shallow trap and then to the oxide conduction band. The transition from direct tunneling to trap-assisted tunneling is shown in Fig. 6. In this figure, the data from Fig. 4 is replotted. The irradiation was at room temperature and the electron detrapping was at $40 \mathrm{~K}$. The total detrapping of the deeper trap is normalized for the four oxide annealing temperatures. As the annealing temperature is increased and the density of shallow traps increases, the field dependence of the electron detrapping changes. At 1100 and $1175^{\circ} \mathrm{C}$, the normalized detrapping is identical. This is because for the $400 \mathrm{~nm}$ buried oxides annealed at $1175^{\circ} \mathrm{C}$ or lower, the concentration of shallow traps is too low to observe trap-assisted tunneling. At $1250^{\circ} \mathrm{C}$, there is a comparable amount of direct and trap-assisted tunneling.

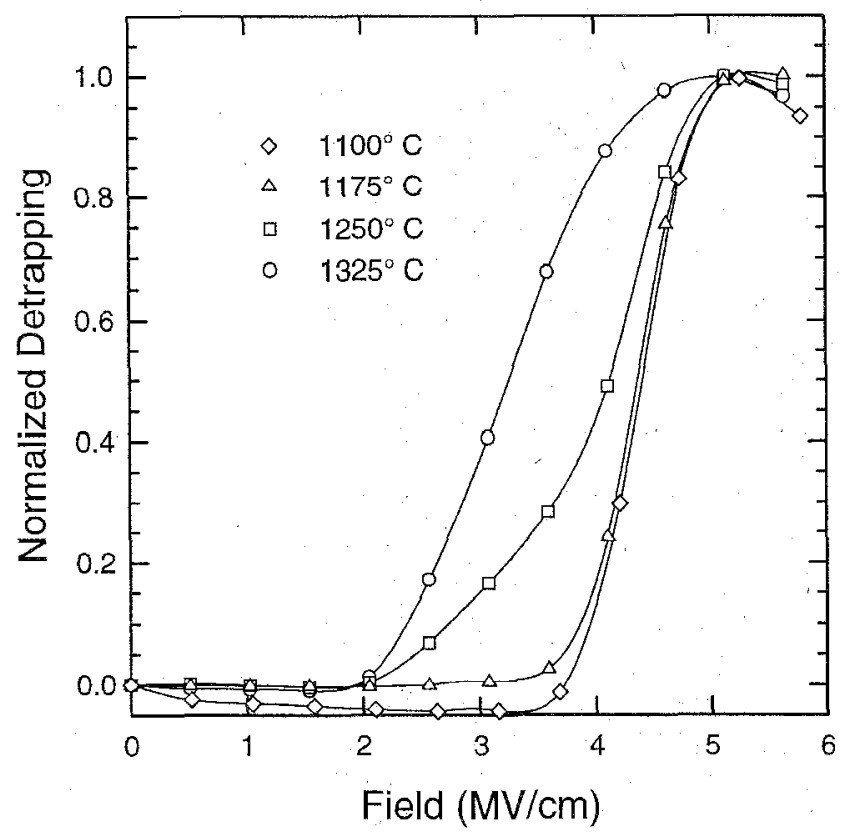

Fig. 6. Normalized field-induced electron detrapping at $40 \mathrm{~K}$ from the deeper electron trap. Results from the $400 \mathrm{~nm}$ buried oxide with annealing temperatures from 1100 to $1325^{\circ} \mathrm{C}$ are shown. Electron (and hole) traps are populated by a radiation dose of $100 \mathrm{krad}\left(\mathrm{SiO}_{2}\right)$ with the capacitor gates grounded at room temperature.

The trap-assisted tunneling starts at $2 \mathrm{MV} / \mathrm{cm}$ while the direct tunneling starts at $3.5 \mathrm{MV} / \mathrm{cm}$. The figure shows that about half of the detrapping is trap assisted and half is direct. That is, half of the deeper traps have a shallow trap that is physically located closer than their tunneling distance. The oxides annealed at $1325^{\circ} \mathrm{C}$ have a higher concentration of shallow traps and most of the detrapping of the deeper traps is by trap-assisted tunneling. At the field threshold for direct tunneling, most of the electrons in the $1325^{\circ} \mathrm{C}$ oxide have already been removed by trap-assisted tunneling.

Note that Eq. 2 was used to calculate the tunneling depth of the deeper trap and that this equation is for trap-to-band tunneling. Using the field-induced detrapping results from the 1250 or $1325^{\circ} \mathrm{C}$ oxides would underestimate the actual tunneling depth.

At the transition from direct to trap-assisted tunneling the concentration of shallow traps can be estimated. For tunneling, the initial and final states must have the same energy. In trap-to-band tunneling the applied field tilts the oxide conduction band to make the trap and conduction band energies the same. Tunneling distances are typically a few nm because tunneling rates increase exponentially with decreasing tunneling distance. By placing a shallower trap along the path to 
the conduction band, the electron can tunnel a longer distance by first tunneling to the intermediate trap and then to the conduction band. The intermediate trap must not be as deep because the conduction band is tilted by the field. Consider tunneling of the deeper electron trap, $1.5 \mathrm{eV}$ deep, at a field of $3.5 \mathrm{MV} / \mathrm{cm}$. Fig. 6 shows that direct tunneling does not occur at this field but that there is trap-assisted tunneling. The distance to tunnel from a trap with a depth of $1.5 \mathrm{eV}$ and a field of $3.5 \mathrm{MV} / \mathrm{cm}$ is $4 \mathrm{~nm}$. To have trap-assisted tunneling there must be a shallow electron trap within this distance that provides a stepping stone for the electron on its path to the oxide conduction band. The volume in which the shallow trap must be located is less than the volume of a hemisphere with a radius of $4 \mathrm{~nm}$. Assuming a random spatial distribution of traps, yields a concentration of shallow traps greater than $10^{19} / \mathrm{cm}^{3}$. This is a lower limit because not all of the positions within the volume of the $4 \mathrm{~nm}$ hemisphere satisfies the tunneling equal energy requirement.

\section{CONCLUSION}

Electron trapping during irradiation is more important in SOI buried oxides than in thermal oxide even if the buried oxide is initially formed by thermal oxidation. Two distinct electron traps are formed by annealing silicon dioxide buried between silicon layers over the temperature range studied: 1100 to $1325^{\circ} \mathrm{C}$. The thermal trap depths are 0.5 and $1.1 \mathrm{eV}$, and the tunneling depths are 0.9 and $1.5 \mathrm{eV}$. Room temperature irradiation populates only the deeper trap and this trap is thermally emptied between room temperature and $120^{\circ} \mathrm{C}$. The maximum electron trapping at room temperature is observed in the $400 \mathrm{~nm}$ thick buried oxide near $1200^{\circ} \mathrm{C}$ and in the $100 \mathrm{~nm}$ thick buried oxides at $1100^{\circ} \mathrm{C}$ (or lower). In buried oxides annealed at higher temperatures the concentration of the shallow electron traps increases and trapping in the deeper electron trap decreases. While electrons are not captured in the shallow trap at room temperature, the high concentration of shallow traps in buried oxides annealed at $1250^{\circ} \mathrm{C}$ and higher leads to trap-assisted tunneling from the deeper traps.

\section{ACKNOWLEDGMENTS}

The author thanks G. A. Brown of Texas Instruments for fabricating the samples and E. S. Steigerwalt at the Naval Research Laboratory for assisting with the data collection.

\section{REFERENCES}

[1] R. E. Stahlbush, W. E. Carlos, and S. M. Prokes, "Radiation and Processing Induced Effects in SIMOX: a Spectroscopic Study," IEEE Trans. Nucl. Sci., vol. NS34, p. 1680, 1987.

[2] H. E. Boesch, Jr., T. L. Taylor, L. R. Hite, and W. E. Bailey, "Time Dependent Hole and Electron Trapping Effects in SIMOX Buried Oxides," IEEE Trans. Nucl. Sci., vol. NS-37, p. 1982, 1990.

[3] W. C. Jenkins, H. L. Hughes, and M. Simons, "Characterization of Back-Channel Leakage-Current Effects in SIMOX Structures at Early Times Following Pulsed Irradiations," J. Rad. Eff. Res. Eng., vol. 9, p. 9, 1990.

[4] C. A. Pennise and H. E. Boesch, Jr., "Determination of the Charge-Trapping Characteristics of Buried Oxides using a 10-keV X-ray Source," IEEE Trans. Nucl. Sci., vol. NS-37, p. 1990, 1990.

[5] A. Stesmans, R. A. B. Devine, A. G. Revesz, and H. L. Hughes, "Irradiation-Induced EPR Active Defects in SIMOX Structures," IEEE Trans. Nucl. Sci., vol. NS-37, p. 2008, 1990.

[6] M. E. Zvanut, R. E. Stahlbush, W. E. Carlos, H. L. Hughes, R. K. Lawrence, R. Heavy, and G. A. Brown, "SIMOX with Epitaxial Silicon: Point Defects and Positive Charge," IEEE Trans. Nucl. Sci., vol. NS-38, p. 1253, 1991.

[7] J. Conley, P. M. Lenahan, and P. Roitman, "Electron Spin Resonance Study of E' Trapping Centers in SIMOX Buried Oxides," IEEE Trans. Nucl. Sci., vol. NS-38, p. 1247, 1991.

[8] R. E. Stahlbush, G. C. Campisi, J. B. McKitterick, W. P. Maszara, P. Roitman, and G. A. Brown, "Electron and Hole Trapping in Irradiated SIMOX, ZMR and BESOI Buried Oxides," IEEE Trans. Nucl. Sci., vol. NS-42, p. 2086, 1992.

[9] J. F. Conley and P. M. Lenahan, "Electron Spin Resonance of Separation by Implanted Oxygen Oxides: Evidence for Structural Change and a Deep Electron Trap," Appl. Phys. Lett., vol. 60, p. 2889, 1992.

[10] W. L. Warren, M. R. Shaneyfelt, J. R. Schwank, D. M. Fleetwood, P. S. Winokur, R. A. B. Devine, W. P. Maszara, and J. B. McKitterick, "Paramagnetic Defect Centers in BESOI and SIMOX Buried Oxides," IEEE Trans. Nucl. Sci., vol. NS-40, p. 1755, 1993.

[11] R. E. Stahlbush, H. L. Hughes, and W. A. Krull, "Reduction of Charge Trapping and Electron Tunneling in SIMOX by Supplemental Implantation of Oxygen," IEEE Trans. Nucl. Sci., vol. NS-40, p. 1740, 1993. 
[12] G. A. Brown and A. G. Revesz, "Defect Electrical Conduction in SIMOX Buried Oxides," IEEE Trans. on Elec. Dev., vol. ED-40, p. 17000, 1993.

[13] V. V. Afanas'ev, A. G. Akos, G. A. Brown, and H. L. Hughes, "Deep and Shallow Electron Trapping in the Buried Oxide Layer of SIMOX Structures," J. Electrochem. Soc., vol. 141, p. 2801, 1994.

[14] R. A. B. Devine, W. L. Warren, J. B. Xu, I. H. Wilson, P. Paillet, and J.-L. Leray, "Oxygen Gettering and Oxide Degradation during Annealing of $\mathrm{Si} / \mathrm{SiO}_{2} / \mathrm{Si}$ Structures," J. Appl. Phys, vol. 77, p. 175, 1995.

[15] R. E. Stahlbush and G. A. Brown, "Bulk Trap Formation by High Temperature Annealing of Buried Thermal Oxides," IEEE Trans. Nucl. Sci., vol. NS-42, p. 1708, 1995.

[16] D. M. Fleetwood, P. S. Winokur, R. A. Reber, Jr., T. L. Meisenheimer, J. R. Schwank, M. R. Shaneyfelt, and L. C. Riewe, "Effects of Oxide Traps, Interface Traps, and "Border Traps" on Metal-Oxide-Semiconductor Devices," J. Appl. Phys., vol. 73, p. 5058, 1993.

[17] R. E. Stahlbush, "Slow and Fast State Formation Caused by Hydrogen," in The Physics and Chemistry of $\mathrm{SiO}_{2}$ and the $\mathrm{Si}_{-} \mathrm{SiO}_{2}$ Interface - 3, H. Z. Massoud, E. H. Poindexter, and C. R. Helms, Eds.: The Electrochemical Society, 1996.

[18] J. G. Simmons and M. C. Tam, "Theory of Isothermal Currents and the Direct Determination of Trap Parameters in Semiconductors and Insulators Containing Arbitrary Trap Distributions," Phys. Rev. B, vol. 7, p. 3706, 1973.

[19] D. M. Fleetwood, S. L. Miller, R. A. Reber, Jr., P. J. McWhorter, P. S. Winokur, M. R. Shaneyfelt, and J. R. Schwank, "New Insights into Radiation-Induced OxideTrap Charge through Thermally-Stimulated-Current Measurements and Analysis," IEEE Trans. Nucl. Sci., vol. NS-39, p. 2192, 1992.

[20] S. E. Thompson and T. Toshikazu, "Tunneling and Thermal Emission of Electrons from a Distribution of Shallow Traps in $\mathrm{SiO}_{2}$," Appl. Phys. Lett., vol. 58, p. $1262,1991$.

[21] C. T. Sah, "Electronic Processes and Excess Currents in Gold-Doped Narrow Silicon Junctions," Phys. Rev., vol. 123, p. 1594, 1961. 